\title{
Gallid Alphaherpesvirus 2 in the Egyptian Turkeys: Molecular Characterization and Establishment of a Universal System for Phylogenetic Classification
}

\author{
Mahmoud Bayoumi ${ }^{\mathrm{a}, \mathrm{b}} \quad$ Mohamed El-Saied $^{\mathrm{c}}$ Basem Ahmed $^{\mathrm{a}}$ Magdy El-Mahdy ${ }^{\mathrm{c}}$ \\ Haitham Amer ${ }^{\text {a }}$ \\ aVirology Department, Faculty of Veterinary Medicine, Cairo University, Giza, Egypt; ${ }^{b}$ Division of Biomedical and Life \\ Sciences, Faculty of Health and Medicine, Lancaster University, Lancaster, UK; 'Pathology Department, Faculty of \\ Veterinary Medicine, Cairo University, Giza, Egypt
}

\section{Keywords}

Egypt · Gallid alphaherpesvirus-2 - Marek's disease · Meq gene $\cdot$ Phylogenetic classification $\cdot$ Turkey $\cdot$ Universal system

\begin{abstract}
Introduction: Gallid alphaherpesvirus 2 (GaHV-2) is a highly contagious oncogenic virus that causes Marek's disease in chickens and occasionally in turkeys. Among 100 genes identified in GaHV-2 genome, the Meq gene appears to involve viral virulence, oncogenicity, and genetic diversity. Despite the use of Meq gene sequences in phylogenetic classification of GaHV-2 strains circulating in many countries worldwide, no integrated system exists yet. Methods: Turkeys from 2 commercial Egyptian farms were presented with signs of dullness, dehydration, and emaciation. Samples prepared from the internal organs were examined by histopathology and immunohistochemistry. Pools of the internal organs were analyzed by PCR for identification of GaHV-2, avian leucosis virus, and reticuloendotheliosis virus. The Meq gene of an Egyptian strain was sequenced and analyzed in comparison to 40 reference strains for generation of a universal system for phylogenetic classification of GaHV-2 strains. Results: Gross and histopathological examination re-
\end{abstract}

karger@karger.com www.karger.com/int

(c) 2021 S. Karger AG, Basel

Karger" vealed grayish-white soft masses in the internal organs characterized by diffuse infiltration of pleomorphic neoplastic cells. All lymphoma cells were identified as T-lymphocytes of CD3+ phenotype. Samples of both farms were only positive for GaHV-2 by PCR. Sequence analysis of the Meq gene has classified the current turkey strain as related to the Egyptian strains identified in chicken in 2012. A universal phylogenetic system for classification of GaHV-2 strains into 4 clusters was proposed. The vaccine strains were all grouped in cluster 2, and most of the classical American strains belonged to cluster 4. Cluster 1 was further divided into 3 subclusters (1.1-1.3). Conclusion: GaHV-2 was identified in turkeys for the first time in Africa and the Middle East. Sequence analysis of the Meq gene of the Egyptian strain along with a wide array of the global strains has enabled the construction of a novel phylogenetic classification system.

(c) 2021 S. Karger AG, Basel

\section{Introduction}

Marek's disease (MD) is one of the most economically devastating infectious diseases of poultry worldwide. The disease principally affects chickens and infrequently involves turkeys [1-3]. However, reports of MD in turkeys 
are increasing in the last few years $[4,5]$. It causes high mortalities among chickens of different age groups, decreased performance, and immune suppression with increasing evidence of vaccination failure [6-8]. MD is caused by Gallid alphaherpesvirus 2 (GaHV-2), which is the prototype member of genus Mardivirus of subfamily Alphaherpesvirinae, family Herpesviridae, and order Herpesvirales $[9,10]$.

GaHV-2 is an oncogenic highly contagious virus that was classified into 3 serotypes based on antigenic and genetic variations. Serotype 1 (prototyped by CVI988/ RISPENS strain) is mostly used as an efficacious live vaccine against MD [10]. Besides, pathogenic strains (mild, virulent, very virulent, and very virulent plus) causing MD are also included $[4,11,12]$. Likewise, serotype 2 (prototyped by SB-1 strain) was used for immunization alone or in combination with MDV serotype $3[13,14]$. Serotype 3 (turkey herpesvirus; HVT) is nonpathogenic to both chickens and turkeys and is frequently utilized as a heterologous live vaccine against other serotypes [10, $15,16]$. The virus primarily replicates in both B- and Tcells (early cytolytic infection), and later establishes a latent infection in CD4+ T-lymphocytes, inducing T-cell lymphoma in visceral organs, skin, and peripheral nerves $[9,10]$.

The genetic basis of GaHV-2 pathogenicity, virulence, tumorigenicity, and host range are not yet fully understood $[17,18]$. The viral genome encodes for $>100$ proteins, among which Marek's EcoQ (Meq) is a basic leucine zipper (b-ZIP) that has the characteristics of a potent transactivator oncoprotein $[19,20]$. Meq protein is the strongest candidate in the protein array involved in T-cell transformation caused by serotype I viruses [21]. Mutation in the Meq gene has confirmed its role as a potential determinant of virus virulence and oncogenicity $[11,12$, 22]. The presence of certain motifs in the transactivation domain of the Meq protein has been reported to associate with the virus pathogenicity in chickens. For instance, the presence of 4 consecutive prolines (PPPP motif) was suggested to correlate with the low pathogenicity of vaccine strains, which have at least 7 PPPP motifs $[11,12]$. These evolutionary markers are valuable tools for prediction of the pathogenicity of MDV strains, considering that the in vivo pathotyping of the ever-rising MDV strains in specific-pathogen-free chicks has limited practicality due to several technical and monetary issues.

Meq gene is also associated with considerable genetic diversity and is mostly utilized as a preferred target for phylogenetic studies [23]. Despite the availability of several phylogenetic classifications for GaHV-2 strains cir- culating in many countries worldwide $[8,9,18,23,24]$, a number of limitations still exist including (1) lack of representation of turkey isolates (2) use of short inconsistent regions of Meq gene (3) random inclusion of viral strains (4) application of different system for nomenclature of strains used in analysis. These limitations precluded the generation of a universal system for the classification of GaHV-2 strains circulating globally.

MD outbreaks have been documented in both vaccinated and unvaccinated chicken flocks from different regions all over the world $[7,9,17,20,25,26]$. The disease was also incriminated to induce sporadic outbreaks in turkeys in Europe [4, 27-30], USA [5], and Asia [2]. No data are available on the circulation of GaHV-2 in turkey in Africa and the Middle East. The genetic characteristics of the turkey isolates remain to be identified. In this study, GaHV-2 was identified in turkey farms located in 2 Egyptian provinces in 2018 . The virus identity was confirmed by histopathology, immunohistochemistry, PCR, and DNA sequencing. Additionally, a universal system for phylogenetic classification of GaHV-2 strains was proposed for further validation.

\section{Materials and Methods}

\section{Clinical Specimens}

Turkeys from 2 commercial farms located in Fayoum and Minia governorates in Egypt (95 and 135 days old, respectively) were presented with signs of depression, dehydration, and a decrease in body weight. Guidelines for sample collection and animal use in research were followed according to the Institutional Animal Care and Use Committee, Faculty of Veterinary Medicine, Cairo University. Samples were collected from the liver, kidneys, spleen, proventriculus, pancreas, sciatic nerves, and intestine of dead birds (10 birds for each farm). The tissue specimens were divided into 2 portions; 1 was fixed for histopathological examination and the second was kept frozen at $-80^{\circ} \mathrm{C}$ till use in genetic analyses.

\section{Histopathological Examination}

Tissue samples were prepared for histopathological examination by fixation in $10 \%$ neutral buffered formalin, rinsing in distilled water, dehydration in ascending grades of methanol, clearance in xylene, and finally embedding in paraffin. Tissues were cut into $4-\mu \mathrm{m}$-thick sections and stained with hematoxylin and eosin. The images were captured at different magnification powers: 40 , 200, 400, and $600 \times$ using an Olympus BX43 microscope equipped with an Olympus digital camera (Olympus, Tokyo, Japan).

Characterization of Neoplastic Cells

The neoplastic cells were determined by immune-histochemical technique using an EnVision ${ }^{\mathrm{TM}}$ FLEX detection system (Dako, Santa Clara, CA, USA) according to the manufacturer's guidelines. In brief, the paraffin-embedded sections were dewaxed and rehydrated, and the epitopes were exposed by heat-induced epitope 
Table 1. Oligonucleotides used for PCR identification of tumor viruses

\begin{tabular}{llcc}
\hline Primers & Sequence $\left(5^{\prime}-3^{\prime}\right)$ & Fragment size (bp) & Reference \\
\hline GaHV2-F & GGATCGCCCACCACGATTACTACC & 313 & {$[32]$} \\
GaHV2-R & ACTGCCTCACACAACCTCATCTCC & 1,020 & {$[7]$} \\
GaHV2 Meq-F & ATGTCTCAGGAGCCAGAGCCG & 436 & {$[5]$} \\
GaHV2 Meq-R & GGGTCTCCCGTCACCTGG & & {$[31]$} \\
ALV-F & AATTCTGCTTGAAATATG & 200 & 855 \\
ALV-R & AGTTGTCAGGGAATCGA & & \\
REV LTR-F & GCGCTGGCTCGCTAACTG & & \\
REV LTR-R & TTCGATCTCGTGTTTTTCGTGATT & & \\
REV env-F & CCACCGGGTCAATAGATGTCAACTG & & \\
REV env-R & AGTGGCTTGTACTGCGGGACTAATG & & \\
\hline
\end{tabular}

bp, base pair; GaHV2, Gallid alphaherpesvirus 2; Meq, Marek’s EcoQ; ALV, avian leucosis virus; REV, reticuloendotheliosis virus.

retrieval through PT-link apparatus as described in [31]. The endogenous peroxidases were blocked by incubation in peroxidase blocking reagent. The tissue sections were incubated overnight with primary mouse antibodies against CD3 and CD20 as markers for T- and B-lymphocytes, respectively [17]. After washing, tissues were incubated for $1 \mathrm{~h}$ with dextran coupled with horseradish peroxidase and goat anti-mouse antibodies. The immune reactivity was revealed by the application of $3,3^{\prime}$-diaminobenzidine tetrahydrochloride and counterstaining with Mayer's hematoxylin. The positive reaction was indicated as a brown stain under the light microscope.

\section{PCR Identification of Tumor Viruses}

Total DNA was extracted from the different tissue samples using the GF-1 total genomic DNA extraction kit (Vivantis, Malaysia) according to the manufacturer's protocol. GaHV-2 was identified in the DNA extract by amplification of the partial ICP4 gene sequence using GaHV2-F and GaHV2-R primers and was confirmed by amplification of the entire Meq gene sequence using GaHV-2 Meq-F and GaHV-2 Meq-R primers (Table 1). The proviral DNA of avian leucosis virus (ALV) and reticuloendotheliosis (REV) was identified using ALV-F and ALV-R, and REV LTR-F and REV LTR-R primers, respectively (Table 1). Further confirmation of REV was achieved by amplification of an 855-bp fragment of the env gene using REV env-F and REV env-R (Table 1). The amplification was performed using EmeraldAmp GT PCR Master Mix (Takara, Kusatsu, Japan) in Gene-Amp 9700 thermal cycler (Applied Biosystems, Foster City, CA, USA). PCR was conducted as follows: initial denaturation at $95^{\circ} \mathrm{C}$ for $5 \mathrm{~min}, 40$ cycles at $95^{\circ} \mathrm{C}$ for $30 \mathrm{~s}, 55^{\circ} \mathrm{C}$ for $30 \mathrm{~s}\left(47^{\circ} \mathrm{C}\right.$ in $\mathrm{ALV}$ and $57^{\circ} \mathrm{C}$ in REV $)$, and $72^{\circ} \mathrm{C}$ for $1 \mathrm{~min}$, and a final extension at $72^{\circ} \mathrm{C}$ for $10 \mathrm{~min}$. Specific PCR products were separated by electrophoresis in $1 \%$ agarose gel stained with ethidium bromide, visualized by a Gel Doc XR gel documentation system (BioRad Laboratories, Milan, Italy), and purified using the Gel/PCR DNA Fragments Extraction Kit (Geneaid, Taiwan)

DNA Sequencing and Phylogenetic Analysis

The purified PCR product of the GaHV-2 Meq gene of a single sample pool, designated as Fayoum-1 2018, was sequenced on both strands using the specific PCR primers at Macrogen Inc (Seoul, South Korea). Contigs were edited and assembled using Bioedit program, version 7.2.5 (Ibis Biosciences, Carlsbad, CA, USA), and the final sequence was deposited in GenBank with the accession number MK261778. A total of $40 \mathrm{Meq}$ gene sequences were retrieved from GenBank for sequence and phylogenetic studies. Multiple alignment of the corresponding sequences was performed using Clustal W algorithm of the MegaAlign program (DNAStar, Madison, WI) for analysis of percent identity, divergence, identification of mutation hotspots, and prediction of amino acid alterations. The proline $(\mathrm{P})$ content and number of PPPP motifs located in the Meq protein were also evaluated. The phylograms were constructed using the maximum-likelihood method in MEGA 6.0 software. Bootstrapping was calculated at 1,000 replicates to confirm the robustness of the phylogenetic trees.

\section{Results}

\section{Gross and Microscopic Lesions}

Postmortem examination of dead turkeys has revealed the presence of soft masses in the majority of internal organs. Tumors of kidneys and liver appeared focal in distribution, grayish white in color with variablesized neoplasms scattered on renal lobes and hepatic surface (Fig. 1a, b). Spleen appeared larger in size (at least twice) with rounded borders and grayish coloration (Fig. 1c). In some examined cases, the spleen showed a mottled appearance. Enlargement in sciatic nerves was remarkable. Histopathological examination showed lymphomatous infiltration of small- to large-sized pleomorphic populations of neoplastic cells with hyperchromatic and mitotic activities in visceral organs and sciatic nerves (Fig. 1d-g). The neoplastic cells appeared multifocal and diffuse in distribution with effaced and re- 


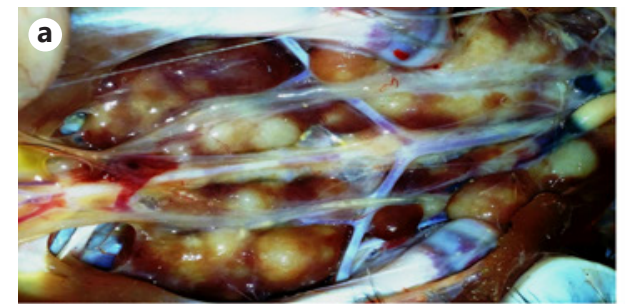

b
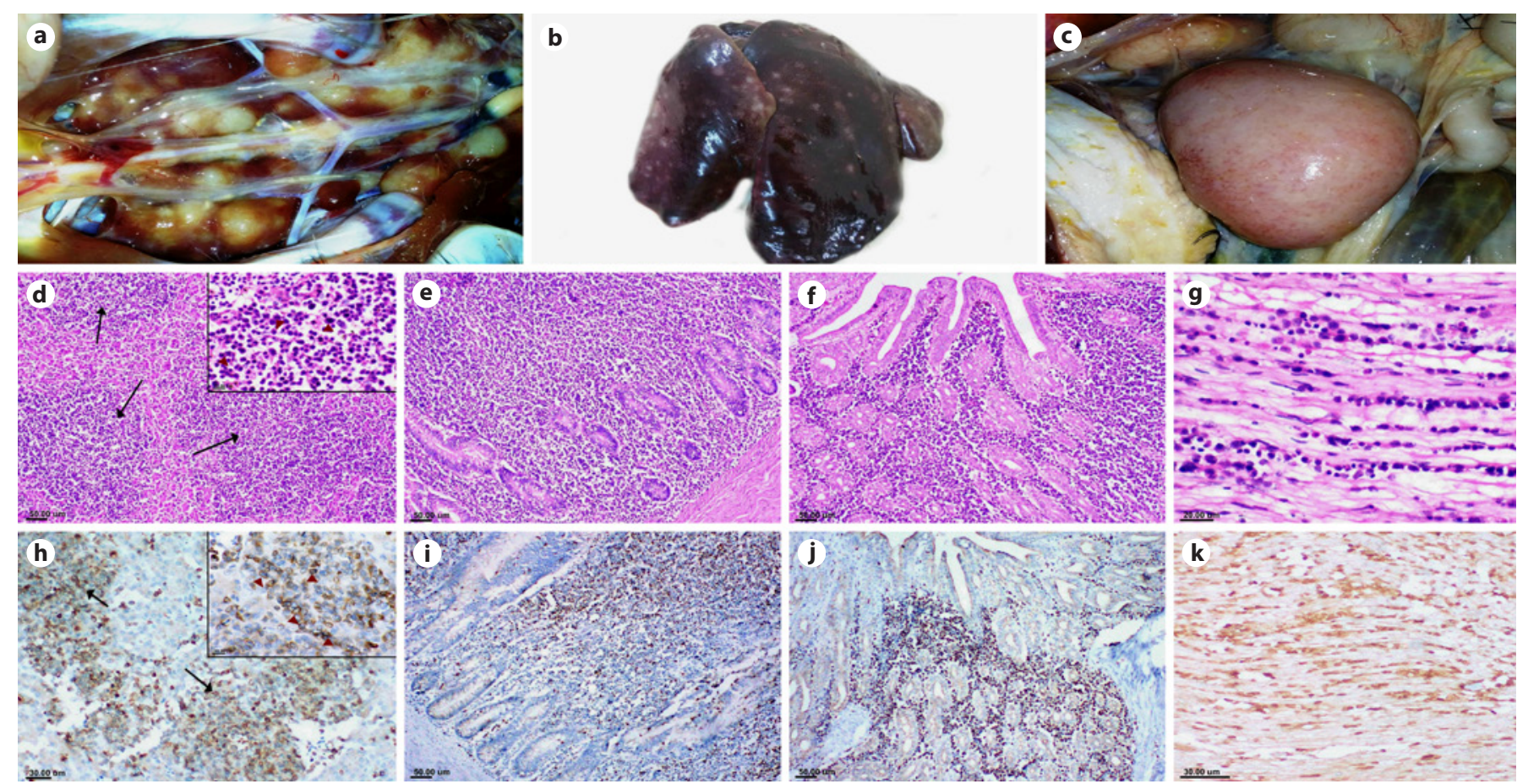

Fig. 1. Photomicrographs showing gross and microscopic representation of Marek's disease lesions in different internal organs of turkeys. Bilateral multifocal variable-sized grayish white foci on renal lobes of the kidneys (a), multifocal discrete white foci in the liver (b), diffuse enlargement in spleen (c), multifocal infiltration of neoplastic lymphoid cells in the liver (black arrows) that replaced the hepatic parenchyma. The inset shows pleomorphic neoplastic cells with mitotic figures (red arrowheads) (d), diffuse infiltration of mixed lymphocyte populations in the lamina propria of the duodenum (e), neoplastic lymphocytic aggregations be-

placed hepatic parenchyma with various necrobiotic changes (Fig. 1d). Furthermore, pleomorphic neoplastic cells were infiltrated between renal tubules and pancreatic islets causing distortion of architecture and atrophy. Moreover, intestinal lamina propria was thickened with diffuse infiltration of heterogenic lymphocytes (Fig. 1e). Additionally, proventricular lobules were infiltrated with a dense population of pleomorphic lymphoid cells between glands (Fig. 1f). Notably, sciatic nerve fibers showed the characteristic type-B nerve lesion that was disrupted by lymphoplasmacytic cell infiltration combined with cell edema and demyelination (Fig. 1g). Immunohistochemical staining identified all lymphoma cells as belonging to the $\mathrm{CD} 3+$ phenotype and negative for CD20. CD3-positive staining was distributed over the sections of neoplastic cells infiltrating the liver (Fig. 1h), intestine (Fig. 1i), proventriculus (Fig. 1j), and sciatic nerve (Fig. 1k). tween the glands of the proventriculus (f), type-B nerve lesion in the sciatic nerve with marked infiltration of pleomorphic neoplastic cells, inflammatory lymphocytes, edema, and demyelination (g), immunohistochemical staining using anti-CD3 antibodies in the liver showing multifocal distribution of neoplastic T-lymphocytes (black arrows) (h), with cytoplasmic reactivity (red arrowheads, insert). Immunohistochemical staining using anti-CD3 antibodies showing positive reactivity of the neoplastic lymphocytic cells in the intestine (f), proventriculus $(\mathbf{j})$, and sciatic nerve (k).

Identification of the Viral Cause of Neoplastic Lesions

Pools of the internal organs of both turkey farms were analyzed for 3 possible tumor-causing viruses of poultry, namely, GaHV-2, REV, and ALV, using PCR. GaHV-2 was identified in both sample pools using serotype-specific primers that target a 313-bp fragment of the ICP4 gene. Positive amplification of both samples was verified against serotype I GaHV-2 (Rispens CVI 988 strain) as a positive control and serotype III GaHV-2 (HVT) as a negative control. The entire sequence of the Meq gene was further amplified to differentiate between oncogenic and nononcogenic strains of GaHV-2. The 2 samples produced PCR amplicons of 1,020 bp (an indicator of oncogenic strains), while the nononcogenic Rispens strain generated a longer band of 1,200 bp. Although samples were also positive for REV by amplification of the LTR fragment, no such amplification was demonstrated with PCR targeting the env gene. All samples were negative for ALV.

Intervirology 2021;64:156-164 
Fig. 2. Phylogenetic analysis of Marek's disease virus strains using the ML method in MEGA 6.0 program. The name, origin, date of identification, and accession number of each strain are indicated. The Egyptian strain identified in this study is marked by a black bullet. Color bars at the right side of the phylogram show the proposed clusters and subclusters. Bootstrap probabilities are denoted at the branch notes. The scale bar at the bottom indicates nucleotide substitutions per site. ML, maximum-likelihood.

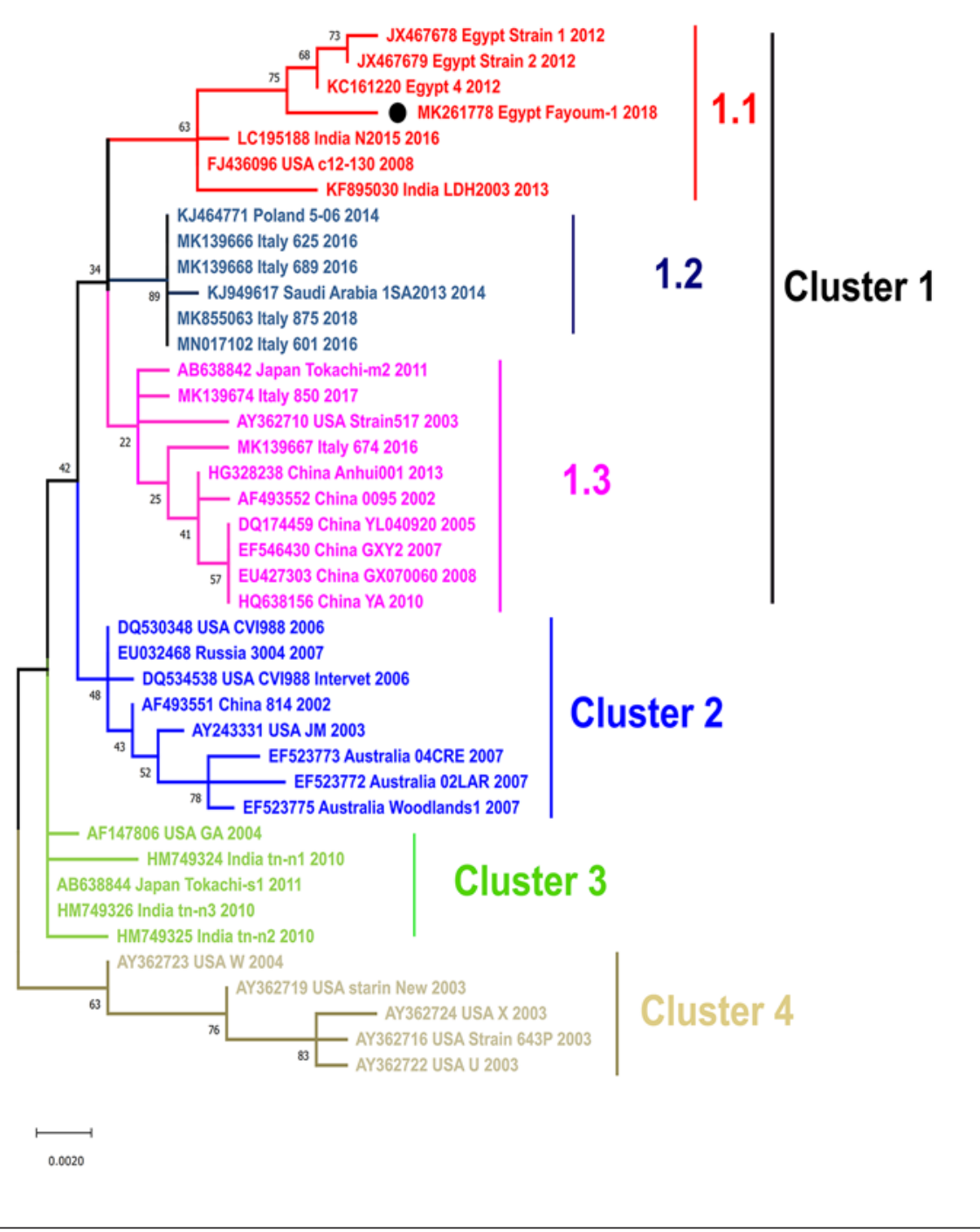

Phylogenetic Analysis and Genotypic Clustering of GaHV-2 Strains

In a trial to unify the genotypic clustering of GaHV-2 globally, a collection of 40 reference strains identified from chickens and turkeys over a diverse of spatial and temporal ranges was included in the sequence and phylogenetic analysis. These strains were chosen to represent the different phylogenetic clusters and pathotypes described before $[8,9,17,18,23-25]$. A large fragment of the Meq gene (924 bp) of the reference strains and Fayoum-1 2018 strain was analyzed using the maximumlikelihood method with bootstrapping of 1,000 replicates. The phylogenetic classification has indicated the separation of GaHV-2 strains into 4 clusters: clusters 1-4 (Fig. 2). The vaccine strains (e.g., CVI988, 3004, 814) were all grouped in cluster 2, whereas the Indian and Japanese strains identified in 2010 and 2011 were members of cluster 3, and most of the classical American strains belonged to cluster 4. Cluster 1 was further divided into 3 subclusters (1.1-1.3). The average homology range between members of the same cluster was $98.5-98.9 \%$ and $95.4-$ $97.1 \%$ at the nucleotide and amino acid levels, respectively, while the homology within the subclusters ranged from 98.6 to $99.6 \%$ (nucleotides) and from 96.7 to $99 \%$ (amino acids). All Egyptian strains including Fayoum-1 2018 were grouped within subcluster 1.1 with an overall homology of $99.4-99.6 \%$ and $98.7-99 \%$ for nucleotides and amino acids, respectively. Another turkey strain recently identified in Italy was grouped within subcluster 1.2 . 


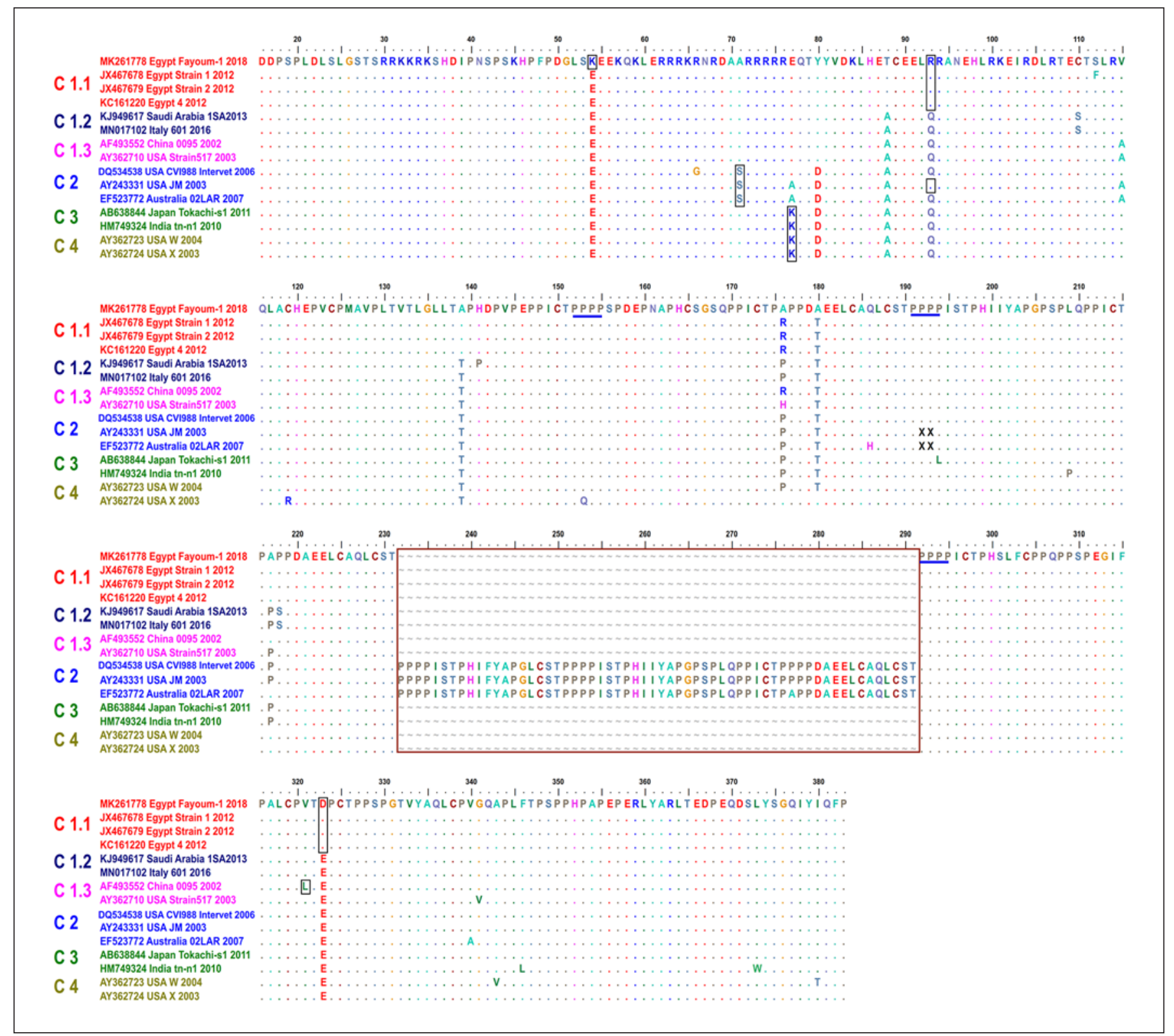

Fig. 3. Deduced amino acid sequence alignment of the MDV Meq protein. The alignment was generated using the ClustalW algorithm of the MegAlign program (DNAstar). The Egyptian strain Fayoum-1 2018 was used as a consensus sequence. Representatives of each proposed cluster and subcluster are indicated on the left side. Identical residues are indicated by dots, and sequence varia-

\section{Molecular Characterization of the Turkey GaHV-2 Meq Protein}

The sequence Fayoum-1 2018 Meq gene/protein was further studied for identification of distinct characteristics. For technical constraints, complete sequence data of the Meq gene was not obtained. Instead, a large fragment

Gallid Alphaherpesvirus 2 in the Egyptian Turkeys tion is denoted by a single-letter code. Black squares identify less common sites, while gaps are dashed. The red rectangle identifies insertion in vaccine strains. The alignment is started from the amino acid residue 16 of the Meq protein. The PPPP motifs are indicated by blue underlines in the Egyptian strain Fayoum-1 2018.

(924 bp) that lacked the first 45 and the last 51 bps of the entire gene was analyzed. Multiple alignment of the partial Meq protein sequence among representative members of the 4 clusters has revealed an overall high degree of sequence conservation. The marked sequence variation was an insertion of a stretch of 60 amino acids ob- 
served in cluster 2 strains, which brings the Meq protein longer in vaccine than in virulent strains. Cluster-specific sites were not observed except for residue 80 (tyrosine in cluster 1 and aspartic acid in other clusters). Likewise, subcluster-specific sites were only evident in subcluster 1.2 that share 2 amino acid substitutions: A88T and Q93R. All Egyptian strains possess a unique amino acid substitution E263D, whereas the turkey strain Fayoum-1 2018 is the only one that shows the mutation record E54K (Fig. 3). To predict the pathogenicity of the identified strain, the PPPP motifs were counted, and only 3 motifs were observed in the Egyptian MDV strains in both chickens and turkeys. Additionally, one interrupted motif from PPPP to PAPP was recorded at position 177 . This interruption characterizes subclusters 1.1 and 1.3 (Fig. 3).

\section{Discussion}

GaHV-2 is an oncogenic herpesvirus that replicates predominantly in T-lymphocytes inducing lymphomatous lesions in chickens and less frequently in turkeys. Despite intensive vaccination and adoption of control measures, outbreaks of MD still occur globally with a significant impact on the economy of the poultry industry $[7,17,18]$. Understanding the genetic diversity among the clinical isolates of GaHV-2 is a key factor that helps determine the causes of vaccination insufficiency to date. Furthermore, the role of turkeys in the epidemiology and evolution of GaHV-2 should be elucidated. In the current study, GaHV-2 strains of turkey origin were identified in 2 commercial farms in Egypt. The polymorphism of the Meq gene as a determinant of virus virulence and tumorigenesis was studied in different GaHV-2 strains identified worldwide over an extended period of time, including the current Egyptian turkey strains.

The major complaint of the 2 turkey flocks was emaciation and uneven growth with a suspect of an oncogenic viral cause. Necropsy of the emaciated turkeys has revealed distinct enlargement of visceral organs with the development of grayish-yellow nodules. These lesions were commonly reported in GaHV-2-infected chickens [9, 17] and turkeys [27, 30]. Enlargement of the sciatic nerve was peculiar in the dissected turkeys. Previous reports only documented infiltration of pleomorphic neoplastic cells in sciatic nerves of turkeys with no evidence of neural lesions $[27,30]$. It seems that the Egyptian strain(s) may have greater pathogenicity and/ or tumorigenicity. Further pathological and molecular characterization of the Egyptian turkey isolates is re- quired to identify the basis of enhanced neuropathogenicity.

In addition to GaHV-2, 2 avian retroviruses including ALV and REV were reported to infect turkeys displaying similar histopathological alterations [6, 27, 29]. Therefore, a stepwise diagnostic approach was followed to confirm that GaHV-2 is the primary cause of disease syndrome, including histopathological examination, immunohistochemistry, PCR, and DNA sequencing. B-cell lymphomas are caused by ALV [32-34], while GaHV-2 recruits T-cells $[34,35]$. Both types of lymphocytes are infiltrated in neoplastic cell formation by REV [30]. Immunohistochemical analysis of tissue samples in the current study has revealed infiltration of lymphocytes with CD3+ and CD20-phenotype, which is specific for T- but not B-cells (Fig. 1h-k). This result augmented that the observed histopathological findings principally referred to GaHV-2 infection [5, 17, 35].

Oncogenic GaHV-2 infection was further confirmed by PCR amplification of the partial sequence of the ICP4 gene and the entire Meq gene. PCR testing for REV using primers specific for the long terminal repeats (LTR) has generated positive amplification results. Although co-infection between GaHV-2 serotype I and REV has been documented in turkeys $[6,36]$, the use of LTR for the identification of REV is mostly misleading. LTR of REV can be inserted in the fowl-pox genome from vaccine and field strains [37]. Therefore, another PCR was performed to prove the absence of full integration of REV proviral DNA in the turkey genome using a primer set specific for the env gene. The results have proved that GaHV-2 is the sole cause of the disease syndrome.

Cell transformation caused by GaHV-2 is principally linked to the transactivation of the Meq gene $[17,21]$. The length of the Meq gene is variable according to the virulence and pathogenicity of the involved strain. Vaccine strains (CVI988, 814, and 3004) and those that induce mild symptoms (e.g., CU-2) have 60 amino acid (aa) longer than virulent strains. Insertion of a stretch of 60 aa residues may have a suppressive effect on Meq gene expression in vaccine and mild strains [9]. The presence of PPPP motifs in the transactivation domain was also linked to the virus pathogenicity. The higher number of the PPPP motif in a given strain is associated with the reduced virulence in affected chicken $[11,12,25]$. In this report, a shorter Meq gene of 1,020 aa length was identified in Fayoum-1 2018 strain. In addition, 3 PPPP motifs in the transactivation domain were recognized with proline content of $21.1 \%$ (data not shown). When comparing these figures with vaccine strains that display 7 PPPP motifs and $23.6 \%$ proline 
content, it may be predicted that Fayoum-1 2018 strain is a virulent to very virulent field strain as proposed before with similar strains $[12,26]$. Sequence analysis has revealed specific aa residues (T88, R93, D263; Fig. 3) similar to those identified in the Egyptian very virulent strains prevalent in chicken in 2012 [9]. This specific pattern may reflect the potential interspecies transmission of the new turkey strain from chicken, and may explain the enhanced neuropathogenicity; however, further molecular and pathological studies are required to support this hypothesis.

Phylogenetic classification of GaHV-2 based on Meq gene sequences has been described before by many groups. However, no consistent grouping of the strains was accomplished till now. The phylograms either presented unclustered strains [18], clustered strains according to the country of origin [23], or clustered strains in 2 or 3 groups identified by Latin numbers I, II, and III [8, $9,17,24]$. Many strains (e.g., Indian Tn-n strains, Chinese strains GX070060, WS03, and YA) were allocated in different clusters according to the criteria used in the phylogenetic analysis $[8,9]$. To unify the system used for phylogenetic clustering of GaHV-2 strains, all the previously constructed phylograms were analyzed and strains that represent every single cluster were chosen. Special care was taken to cover the entire array of geographical regions, temporal chronology of outbreaks, and different host systems. We also proposed the use of almost the entire Meq gene in the generation of the unified phylogram. It is expected that the phylogenetic classification proposed in this study will provide a platform that will help to understand the epidemiology and evolution of GaHV2 in both chicken and turkeys.

In conclusion, GaHV-2 was recorded in turkeys for the first time in Egypt (and possibly in Africa and the Middle East). The emergence of MD in a new species might pose threats to the poultry industry, particularly in the context of close proximity between the commercial farms of chicken and other avian species in Egypt. The inefficient biosecurity practices adopted in turkey farms compared to that of chicken counterparts provides ample opportu- nities for multispecies transmission and development of outbreaks. Comprehensive epidemiologic and molecular studies are essentially required to explore the prevalence and significance of GaHV-2 in turkeys worldwide.

\section{Acknowledgements}

We would like to thank all colleagues and coworkers in both virology and pathology departments, Faculty of Veterinary Medicine, Cairo University, Egypt, for their technical support.

\section{Statement of Ethics}

Guidelines for sample collection and animal use in research were followed according to the Institutional Animal Care and Use Committee, Faculty of Veterinary Medicine, Cairo University.

\section{Conflicting Interest Statement}

The authors declare that they have no conflicting interests.

\section{Funding Source}

No funding was received for this study.

\section{Author Contributions}

Conceptualization: Haitham Amer and Magdy El-Mahdy. Methodology: Mahmoud Bayoumi and Mohamed El-Saied. Formal analysis and investigation: Haitham Amer, Basem Ahmed, and Magdy El-Mahdy. Writing - original draft preparation: Mahmoud Bayoumi and Mohamed El-Saied. Writing - review and editing: Haitham Amer, Basem Ahmed, and Magdy El-Mahdy. Resources: Mohamed El-Saied. Supervision: Haitham Amer and Magdy El-Mahdy.

\section{References}

1 Buckmaster AE, Scott SD, Sanderson MJ, Boursnell MEG, Ross NLJ, Binns MM. Gene sequence and mapping data from Marek's disease virus and herpesvirus of turkeys: implications for herpesvirus classification. J Gen Virol. 1988;69(8): 2033-42.

2 Davidson I, Malkinson M, Weisman Y Marek's disease in turkeys. I. A seven-year

Gallid Alphaherpesvirus 2 in the Egyptian Turkeys survey of commercial flocks and experimental infection using two field isolates. Avian Dis. 2002;46(2):314-21.

3 Nair V. Spotlight on avian pathology: Marek's disease. Avian Pathol. 2018;47(5): $440-2$.

4 Mescolini G, Lupini C, Davidson I, Massi P, Tosi G, Fiorentini L, et al. Molecular characterization of a Marek's disease virus strain de- tected in tumour-bearing turkeys. Avian Pathol. 2020;49(2):202-7.

5 Hauck R, Mays J, Dunn JR, Shivaprasad HL. Two cases of Marek's disease in backyard Turkeys. Avian Dis. 2020;64(3):347-51.

6 Payne LN, Venugopal K. Neopastic diseases: Marek's disease, avian leukosis and reticuloendotheliosis. Rev Sci Tech l'OIE.. 2000; 19(2):544-64. 
7 Gong Z, Zhang L, Wang J, Chen L, Shan H, Wang Z, et al. Isolation and analysis of a very virulent Marek's disease virus strain in China. Virol J. 2013;10:155-8

8 Puro K, Bhattacharjee U, Baruah S, Sen A, Das S, Ghatak S, et al. Characterization of Marek's disease virus and phylogenetic analyses of meq gene from an outbreak in poultry in Meghalaya of Northeast India. Virus Disease. 2018;29(2):167-72.

9 Hassanin O, Abdallah F, El-araby IE. Molecular characterization and phylogenetic analysis of Marek's disease virus from clinical cases of Marek's disease in Egypt. Avian Dis. 2013;57: $555-61$.

10 Boodhoo N, Gurung A, Sharif S, Behboudi S. Marek' s disease in chickens : a review with focus on immunology. Vet Res. 2016:1-19.

11 Shamblin CE, Greene N, Arumugaswami V, Dienglewicz RL, Parcells MS. Comparative analysis of Marek's disease virus (MDV) glycoprotein-, lytic antigen pp38- and transformation antigen Meq-encoding genes: association of meq mutations with MDVs of high virulence. Vet Microbiol. 2004;102(3-4):14767.

12 Renz KG, Cooke J, Clarke N, Cheetham BF, Hussain Z, Fakhrul Islam AFM, et al. Pathotyping of Australian isolates of Marek's disease virus and association of pathogenicity with meq gene polymorphism. Avian Pathol. 2012; 41(2):161-76.

13 Schat KA, Calnek BW. Protection against Marek's disease-derived tumor transplants by the nononcogenic SB-1 strain of Marek's disease virus. Infect Immun. 1978;22(1):225-32.

14 Witter RL. Increased virulence of Marek's disease virus field isolates. Avian Dis. 1997 JanMar;41(1):149.

15 Witter RL, Solomon JJ. Experimental infection of Turkeys and chickens with a herpesvirus of Turkeys (HVT). Avian Dis. 1972;16(1): 34-44.

16 Afonso CL, Tulman ER, Lu Z, Zsak L, Rock DL. The genome of Turkey herpesvirus. J Virol. 2001;75(2):971-8.
17 Abd-ellatieff HA, Rawash AAA, Ellakany HF, Goda M, Suzuki T, Yanai T. Molecular characterization and phylogenetic analysis of virulent Marek s disease virus field strain in broiler chickens in Japan. Avian Pathol. 2017: $1-41$.

18 Mohamed MHA, El-sabagh IM, Al-habeeb MA, Al-hammady YM. Diversity of Meq gene from clinical Marek's disease virus infection in Saudi Arabia. Vet World. 2016;9:572-8.

19 Jones D, Lee L, Liu JL, Kung HJ, Tillotson JK. Marek disease virus encodes a basic-leucine zipper gene resembling the fos/jun oncogenes that is highly expressed in lymphoblastoid tumors. Proc Natl Acad Sci USA. 1992;89(9): 4042-6.

20 Hartawan R, Dharmayanti NLPI. The Meq gene molecular profile of Marek's disease virus serotype 1 from Kampung and Arabic chicken farms in Sukabumi, West Java, Indonesia. Hayati J Biosci. 2017;23(4):160-7.

21 Ajithdoss DK, Reddy SM, Suchodolski PF, Lee LF, Kung HJ, Lupiani B. In vitro characterization of the Meq proteins of Marek's disease virus vaccine strain CVI988. Virus Res. 2009; 142(1-2):57-67.

22 Woźniakowski G, Samorek-salamonowicz E. Molecular evolution of Marek's disease virus ( MDV ) field strains in a 40-year time period. Avian Dis. 2014;58(Mdv):550-7.

23 Padhi A, Parcells MS. Positive selection drives rapid evolution of the meq oncogene of Marek's disease virus. PLoS One. 2016:1-15.

24 Tian M, Zhao Y, Lin Y, Zou N, Liu C, Liu P, et al. Comparative analysis of oncogenic genes revealed unique evolutionary features of field Marek's disease virus prevalent in recent years in China. Virol J. 2011;8:121-11.

25 Mescolini G, Lupini C, Davidson I, Massi P, Tosi G, Catelli E. Marek's disease viruses circulating in commercial poultry in Italy in the years 2015-2018 are closely related by their meq gene phylogeny. Transbound Emerg Dis. 2020;67(1):98-107.

26 Mescolini G, Lupini C, Felice V, Guerrini A, Silveira F, Cecchinato M, et al. Molecular characterization of the meq gene of Marek's disease viruses detected in unvaccinated backyard chickens reveals the circulation of low- and high-virulence strains. Poult Sci. 2019;98(8):3130-7.
27 Pennycott TW, Venugopal K. Outbreak of Marek's disease in a flock of turkeys in Scotland. Vet Rec. 2002;150:277-9.

28 Deuchande R, Murphy A, Otter A, Baigent S, Wood A, Irvine RM. Marek's disease in Turkeys. Vet Rec. 2012;171(23):602.

29 Blake-Dyke C, Baigent S. Marek's disease in commercial turkey flocks. Vet Rec. 2013; 173(15):376.

30 Hughes K, Archer J, Constantino-Casas F, Wozniakowski GJ, Baigent S. Diagnostic investigation of Marek's disease in a turkey. Vet Rec Case Rep. 2016;4(1):e000291.

31 Bayoumi M, El-Saied M, Haitham A, Mostafa B, Sakr EE, El-Mahdy M. Molecular characterization and genetic diversity of the infectious laryngotracheitis virus strains circulating in Egypt during the outbreaks of 2018 and 2019. Arch Virol. 2020;165(3):661-70.

32 Hayward WS, Neel BG, Astrin SM. Activation of a cellular onc gene by promoter insertion in ALV-induced lymphoid leukosis. Nature. 1981;290(5806):475-80.

33 Justice IVJ, Beemon KL. Avian retroviral replication. Curr Opin Virol. 2013;3(6):664-9.

34 Malhotra S, Winans S, Lam G, Justice J, Morgan R, Beemon K. Selection for avian leukosis virus integration sites determines the clonal progression of B-cell lymphomas. PLoS Pathog. 2017;13(11):e1006708-25.

35 Pejovic N, Maja V, Sanja A-K, DM, Milijana K. Morphological and immunohistochemical examination of tumor cells in Marek's disease. Acta Vet Brno. 2007;57(1):27-35.

36 Davidson I, Borovskaya A, Perl S, Malkinson M. Use of the polymerase chain reaction for the diagnosis of natural infection of chickens and turkeys with Marek's disease virus and reticuloendotheliosis virus. Avian Pathol. 1995; 24:69-94

37 García M, Narang N, Reed WM, Fadly AM. Molecular characterization of reticuloendotheliosis virus insertions in the genome of field and vaccine strains of fowl poxvirus. Avian Dis. 2003 Apr-Jun;47(2):343-54 